\title{
Molecular simulations of the adsorption and diffusion of hydrocarbons in molecular sieves
}

\section{Berend Smit}

Centre Européen de Calcul Atomique Moléculaire (CECAM), Ecole Normale Supérieure, 46 Allée d'Italie, 69364 Lyon Cedex 7, France

\begin{abstract}
In this short review it is demonstrated how molecular simulation can be used to obtain molecular insights in the behaviour of molecules inside the pores of zeolites. Whereas straightforward molecular dynamics can be used to study the behaviour of small molecules inside the pores, the study of the long chain hydrocarbons that are important in petrochemical applications require special computational techniques such as configurational-bias Monte Carlo or rare event simulation techniques. The use of these techniques is illustrated with some examples of practical importance such as the adsorption of mixtures of linear and branched hydrocarbons, and the loading dependence of the diffusion coefficients.
\end{abstract}

\section{INTRODUCTION}

Before a molecule can react inside the pores of a zeolite, it has to adsorb and to diffuse to the active site[1]. Understanding the diffusion and adsorption of molecules inside the pores of a zeolite is therefore an integral part of our understanding of catalyses in zeolite. Many of Barrer's articles have been aimed at this understanding [2].

In this short review it is shown how molecular simulations can complement the experimental work in our understanding of the behaviour of molecules inside the pores of a zeolite. In particular, we address three seemingly simple questions:

- What is the composition of molecules inside the pores of a zeolite?

- Does the diffusion coefficient of a molecule increase or decrease as a function of loading?

- What is the contribution of the zeolite to the free energy of formation of molecules that are formed inside the pores of a zeolite?

From a computational point of view addressing this question is an enormous challenge. Small molecules or atoms, such as the noble gasses or methane can be conveniently studied using molecular simulations, but special techniques have to be used for the long chain hydrocarbons that are of interested for petrochemical applications [3].

This short review illustrates how computational techniques have been evolved such that the questions like the above can be addressed. 


\section{MOLECULAR SIMULATION TECHNIQUES}

The idea of a molecular simulation is simple; provide a model in the form of an intermolecular potential that describes the interaction between the molecules adsorbed in the zeolite. This model provides the input for a molecular dynamics simulation or a Monte Carlo simulation from which the corresponding thermodynamic and/or transport properties are obtained. For the successful application of these techniques it is important that the intermolecular potentials give a sufficiently realistic description of the experimental systems and that the simulation techniques are sufficiently powerful such that, for the molecules of interest, accurate properties can be computed within a reasonable amount of cpu-time.

Ideally, one would like to mimic the real system as closely as possible. If successful, one would also reproduce the experimental data, including the diffusion coefficients. Hence, one would also observe that for the long-chain hydrocarbons that are of interest to hydrocracking, the diffusion coefficient is so small that the corresponding simulations to obtain reliable statistics are getting prohibitively long. As a consequence, standard molecular dynamics can only be used for those systems that diffuse sufficiently fast that accurate thermodynamic or transport properties can be obtained.

Let us consider the experimental setup to measure adsorption isotherms. We would like to measure the number of adsorbed molecules as a function of the pressure of the gas or liquid that is in contact with the zeolite. Experimentally, the most common system is a zeolite in a container that contains a gas or liquid. The liquid or the gas is kept at a constant temperature and pressure or partial pressure in case of a mixture. In equilibrium, the adsorbed gas molecules have the same temperature and chemical potential as the molecules in the container. The container can be seen as a reservoir that fixes the temperature and chemical potentials of the adsorbed components. This experimental setup closely resembles the grand-canonical ensemble [4]. In the grand-canonical ensemble the temperature, volume, and chemical potentials are imposed and we can perform a simulation in which the reservoir and zeolite are not in direct physical contact, but the procedure guarantees that the adsorbed molecules have an equal temperature and chemical potential in both the reservoir and in the zeolite.

Grand-canonical Monte Carlo works best if the acceptance of trial moves by which particles are added or removed is not too low [4]. For atomic fluids, this condition effectively limits the maximum loading in a zeolite at which the method can be used. Although the grand-canonical Monte Carlo technique can be applied to simple models of non-spherical molecules, special techniques are required since the method converges very poorly for all but the smallest polyatomic molecules.

Both molecular dynamics and Monte Carlo are very efficient for atoms or small molecules, but for large molecules both methods require significant amounts of cpu-time. For example, June et al. [5] studied the relaxation of n-butane and n-hexane in MFI using molecular dynamics and concluded that the zeolite slowed down the relaxation of these molecules by several orders of magnitude; the longer the chains, the slower the relaxation. Hence, the cpu-requirements increase significantly for MD simulations of these long-chain alkanes. The diffusion coefficients of linear alkanes in MFI are sufficiently high that these can be simulated using MD [6], but for the mono branched alkanes MD can only be used at very high temperatures $[7,8]$. Branched alkanes in MFI preferentially adsorb in the intersections between the zig-zag and straight channels [9], the diffusion is therefore an activated process in which the molecule jumps from one intersection to another [10].

This very slow diffusion path could be avoided via a Monte Carlo simulation in which we generate a new configuration at a random position in the zeolite. The probability that such 
a move will be accepted depends on the energy difference between the new and the old configuration. Clearly, if we generate a new position on top of a zeolite atom the attempt will be rejected. For a chain molecule this implies that none of the atoms should overlap with the zeolite atoms. If for methane this probability is 1 out of 1000 attempts, for ethane this will be of the order 1 out of $10^{6}$, and for n-octane 1 out of $10^{24}$. The conventional Monte Carlo method is therefore very efficient for noble gasses or small molecules, but for long-chain alkanes it is equally inefficient as molecular dynamics.

The configurational-bias Monte Carlo (CBMC) technique has been developed to make the insertion of long-chain molecules in moderately dense liquids possible. The original configurational-bias Monte Carlo technique was developed for lattice models and has been extended to continuous models [11]. In a CBMC simulation one grows a molecule atom by atom using a method based on an algorithm developed by Rosenbluth and Rosenbluth [12]. In this growing scheme one avoids overlap with the zeolite atoms. The corresponding bias is removed exactly by adjusting the acceptance rules [4].

A very small diffusion coefficient often is the result of molecules trapped in low (free) energy sites and once in a while hops from one to another adsorption site. To compute a diffusion coefficient reliably one has to observe a sufficient number of hops. Most of the cputime is, however, spent on molecules that "wait" close at an adsorption site until a fluctuation gives them sufficient kinetic energy to take the barrier between adsorption sites. The higher the barrier the longer the molecules remain trapped and -on the time scale of a molecular dynamics simulation- such a hopping becomes a very rare event. Special techniques have been developed to simulate such rare events [4]. The basic idea is to compute the hopping rate in two steps $[13,14]$. First, we compute the probability that a molecule can be found on top of the barrier. This calculation is followed by a separate simulation in which the probability is computed that a molecule that starts on top of the barrier end up in the next adsorption site and does not re-cross the barrier.

The probability to find a molecule on top of the barrier can be computed directly from the free energy profile, which is the free energy as a function of the position of the molecule in the zeolite. The second step involves the average time it takes a molecule to cross the barrier. The simplest approach is to assume that transition state theory (TST) holds. A molecule that arrives at the top of the barrier is assumed to be in equilibrium with its surrounding, as a consequence the velocity distribution is given by the Maxwell distribution corresponding to the temperature of the system. TST assumes that half of the molecules that reach the barrier also cross the barrier. TST theory ignores the possibility that such a particle re-crosses the barrier and returns into the cage it originates from due to, for example, collisions with the zeolite atoms. This re-crossing can be computed directly from a molecular dynamics simulation in which the molecules start on top of the barrier and this re-crossing probability is directly computed. As this involves a simulation that starts on top of the barrier, it is much faster than simulating the time it takes a molecule to climb the free energy barrier. These rare-event methods have been applied to zeolites at low [10,15-18] and high loadings [19].

\section{INTERMOLECULAR POTENTIALS}

Most simulation studies follow the assumptions pioneered by Kiselev and co-workers [20] for the adsorption of non-polar molecules. The zeolite is assumed to be rigid and purely siliceous. The adsorbate-zeolite interactions are dominated by the dispersive interactions with the oxygen atoms of the zeolite. The smaller silicon atoms contribute little to the dispersive 
interaction and are taken into account implicitly via the oxygen atoms. Further refinements involve the use of a flexible lattice, or the effect of charges for systems that involve polar or Coulombic interactions [21].

If a rigid lattice is assumed one does not need a model for the zeolite-zeolite interactions. In case a flexible zeolite is essential, various models that describe the zeolite-zeolite interactions have been published. The accuracy of these models can partly be assessed via a comparison of the calculated vibrational IR spectra with the experimental ones. Since these models have been discussed in detail by Demontis and Suffritti [22], we refer to this review for details on these models and further references. To limit the cpu-requirements of a fully flexible zeolite, methods have been developed in which the normal vibrational modes and harmonic crystal approximation are used [23].

For the simulation of hydrocarbons various models have been proposed in the literature. The most realistic models are all-atom models in which both the carbon and hydrogen atoms are considered explicitly. In united-atom models the $\mathrm{CH}_{3}, \mathrm{CH}_{2}$, or $\mathrm{CH}$ groups are considered as a single atom. From a computational point of view the united-atom model is more efficient and has fewer parameters that have to be determined. Therefore, most studies use a unitedatom model. Comparison of the results of such simulations with experimental data for the adsorption and diffusion shows a satisfactory description of the experimental data. In addition, the scatter in these currently available experimental data makes it very difficult to prove that an all-atom model is essential.

For the adsorbate-adsorbate interactions it is convenient to distinguish the intramolecular interactions and the intermolecular interactions. The intramolecular interactions are very important to arrive at a realistic representation of the conformation of the adsorbate molecules. Fortunately, these potentials can be based on quantum chemical or spectroscopic data and therefore for most molecules these models give a sufficiently accurate description of these intramolecular interactions. In addition, comparisons of various models, for example the torsion or bond-bending, show little influence on the thermodynamic properties such as the vapor-liquid curve [24]. For the hydrocarbon special force fields, both united-atom and allatom models have been developed that give an accurate description of the entire vapor-liquid coexistence curve [24-33].

Often the zeolite-adsorbate interaction parameters are obtained from fitting to experimental data, and therefore these parameters depend on which accuracy and type of data are used in the fitting procedure. For example, the parameters obtained from fitting to diffusion coefficients can be different from those fitted to heats of adsorption or Henry coefficients.

Beerdsen et al. have shown that a very accurate fitting of the parameters of a united-atom model can be obtained through fitting on experimental isotherms with inflection points [19]. This procedure uniquely determined the adsorbent-adsorbate interaction parameters and is very sensitive to the size parameter as the inflection points in the isotherms are often related to a subtle interplay between different adsorption sites. Figure 1 shows a typical example of the agreement with experimental data that can be obtained. Several alternative force fields have been developed that also give an accurate description of the experimental adsorption isotherms $[35,36]$. 


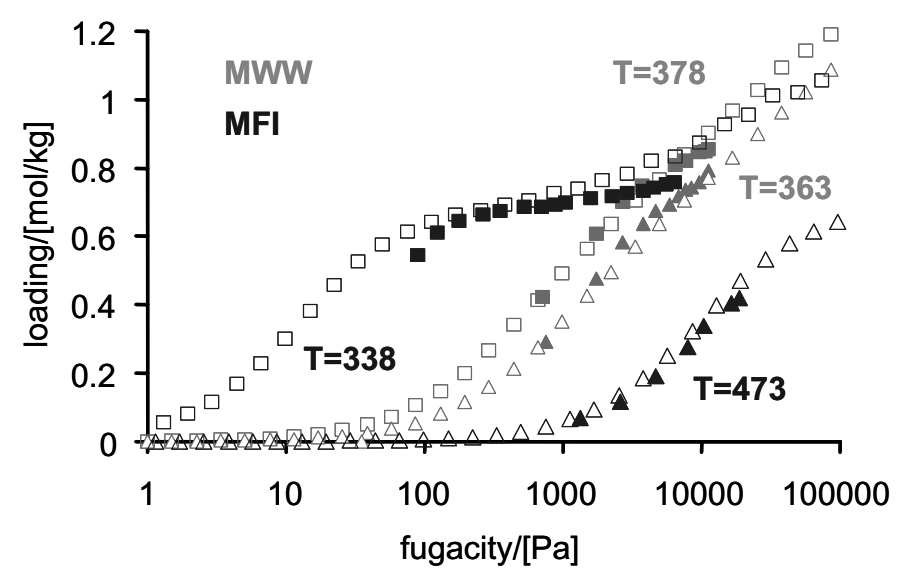

Fig. 1. Comparison between the simulated (open symbols) and experimental (closed symbols) of the adsorption isotherm of n-hexane in MWW and MFI (data from ref. [34]).

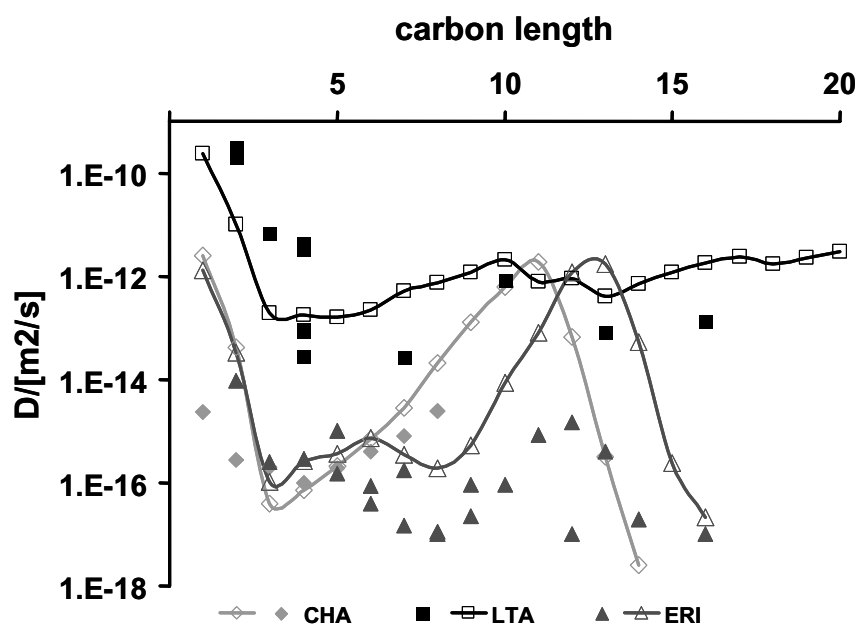

Fig. 2. Comparison of the experimental (closed symbols) and the simulated diffusion coefficients of linear n-alkanes in the zeolites CHA, LTA, and ERI. (The data are taken from ref. [45].)

\section{APPLICATIONS}

\subsection{Adsorption of Mixtures}

Experimentally a pure component isotherm can be measured relatively easily using gravimetrical techniques. However, for mixtures one has to carry out additional measurement to determine the composition of the molecules inside the pores.

A simple example is the adsorption of methane and ethane. On energetic arguments one would expect ethane to be preferentially adsorbed above methane. This is indeed what simulations and experiments show at low pressures[37] in MFI, but at high pressures the selectivity reverses. Entropically it is, however, much more favourable to pack the small 
methane molecules in the pores of the zeolite and hence at high pressure the methane is displacing the bigger ethane molecules from the zeolite. Krishna et al.[38] have reviewed the various entropic effects one can expect in zeolites.

These entropy effects can be very surprising. For example, in for a mixture of linear and branched alkane isomers, the optimal packing at high pressures favours the adsorption of the linear isomers in MFI [39]. In the one-dimensional tube like zeolites such as AFI and TON, depending on the diameter either the branched or the linear isomers are preferentially adsorbed $[40,41]$.

\subsection{Diffusion of Hydrocarbons}

Experimentally it is very difficult to obtain reliable data on the diffusion of hydrocarbons in zeolites[42]. Depending on the experimental technique that has been used the resulting diffusion coefficients may differ by orders of magnitude.

Understanding the diffusion behaviour in zeolites is extremely interesting from a scientific point of view as the zeolite topology may completely change the qualitative diffusion behaviour. Normally one would argue that as the hydrocarbon chain gets longer the diffusion coefficient decreases. This has indeed been observed in most zeolites, but for some zeolites the diffusion coefficient increases with increasing carbon number. This nonmonotonic diffusion behaviour has been observed experimentally many years ago by Gorring [43], but more recent experiments did not provide such indications[44]. Interestingly, computer simulations do support the observations of Gorring (see Figure 2). Hopefully, this dispute will stimulate new experimental work.

For practical applications it is important to understand the dependence of the diffusion coefficient on the loading. For many years it was generally accepted that in zeolites the Darken assumption would hold, i.e. the corrected diffusion coefficient is independent of loading. Recent, molecular simulations of Skoulidas and Sholl [46], however, have shown that this assumption may hold for only a few zeolites. Beerdsen et al have subsequently shown that at sufficiently high loading also for these zeolites the Darken assumption breaks down $[47,48]$.

\subsection{Free Energies of Formation}

Hydroconversion reactions in zeolite involve many competing reaction paths. Text book reaction kinetics states that those paths with the reaction intermediates that have the lowest free energy of formation dominate the reaction. The difficulty in applying these text book results to zeolites is that the contribution of the zeolite to the free energy of formation is unknown. Interestingly, using molecular simulations one can estimate these free energies. This information can be used to obtain some new insights in the reaction mechanisms underlying inverse shape selectivity [40], cage effects [49], or the window effect [50] (see also figure 3 ). 

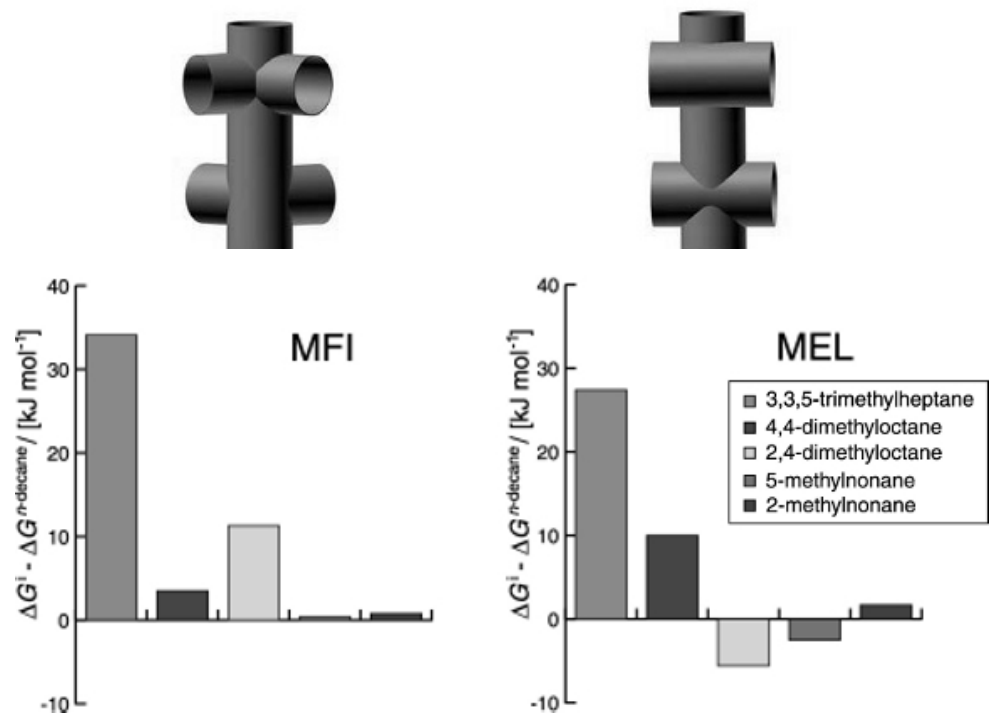

Fig. 3. Contribution of the zeolite to the free energy of formation of some important reaction intermediates in the structures MFI and MEL. In MFI the reaction path is dominated by 4,4 dimethyloctane isomer while in MEL the path is dominated by 2,4 dimethyloctane [51] .

\section{CONCLUDING REMARKS}

In this review we have illustrated how molecular simulations can give us important additional information on the behaviour of molecules inside the pores of a zeolite. Of course, these computations have been carried out on well defined model systems. Many of the results have not yet been confirmed by experiments. Hence, one may conclude they are completely speculative. However, from a computational point of view the techniques have advanced such that, if one assumes that a zeolite and a hydrocarbon can be reasonably described with the models that are discussed on this work, the resulting predictions for the thermodynamic and transport properties can be reliably computed. At this point it is important to mention that these techniques can also be applied to more sophisticated models. Comparison with the available experimental evidence shows that, at present, there is no need to make more accurate models. This observation does not make the simulation results less speculative, but they are the best speculation we can do.

At this point it is important to mention that in our models we assume perfect, defect free crystals in which the boundaries have negligible effects. Real materials are far from ideal and taking into account these non-idealities remains to be done.

\section{ACKNOWLEDGEMENTS}

This review is based on work done in collaboration with many excellent researchers. I am particularly indebted to Edith Beerdsen, Sofia Calero, Theo Maesen, R. Krishna, Thijs Vlugt, 
Merijn Schenk, Bei Liu, and David Dubbeldam. This work is partly supported by the EC via a Marie Curie Excellence Grant (MEXT-CT-2005-023311).

\section{REFERENCES}

[1] J. M. Thomas, Sci. Am. 266 (1992) 82.

[2] R. M. Barrer, Zeolites and clay minerals as sorbents and molecular sieves. ( Academic Press, London, 1978).

[3] Computer modeling of micorporous materials, edited by C. R. A. Catlow, R. A. van Santen, and B. Smit (Elsevier, Amsterdam, 2004).

[4] D. Frenkel and B. Smit, Understanding Molecular Simulations: from Algorithms to Applications, 2nd ed. (Academic Press, San Diego, 2002).

[5] R. L. June, A. T. Bell and D. N. Theodorou, J. Phys. Chem. 96 (1992) 1051.

[6] R. C. Runnebaum and E. J. Maginn, J. Phys. Chem. B 101 (1997) 6394.

[7] D. Schuring, A. P. J. Jansen and R. A. v. Santen, J. Phys. Chem. B 104 (2000) 941.

[8] E. B. Webb, G. S. Grest and M. Mondello, J. Phys. Chem. B 103 (1999) 4949.

[9] T. L. M. Maesen, M. Schenk, T. J. H. Vlugt, J. P. de Jonge and B. Smit, J. Catal. 188 (1999) 403.

[10] T. R. Forester and W. Smith, J. Chem. Soc. Faraday Trans. 93 (1997) 3249.

[11] D. Frenkel, G. C. A. M. Mooij and B. Smit, J. Phys.: Condens. Matter 4 (1992) 3053.

[12] M. N. Rosenbluth and A. W. Rosenbluth, J. Chem. Phys. 23 (1955) 356.

[13] C. H. Bennett, in Algorithms for chemical computations, edited by R. E. Christoffersen (American Chemical Society, Washington, DC, 1977), pp. 63.

[14] D. Chandler, J. Chem. Phys. 68 (1978) 2959.

[15] D. Dubbeldam, S. Calero, T. L. M. Maesen and B. Smit, Angew. Chem. 42 (2003) 3624.

[16] D. Dubbeldam, E. Beerdsen, T. J. H. Vlugt and B. Smit, J. Chem. Phys. 122 (2005).

[17] F. Jousse and S. M. Auerbach, J. Chem. Phys. 107 (1997) 9629.

[18] T. Mosell, G. Schrimpf and J. Brickmann, J. Phys. Chem. B 101 (1997) 9476.

[19] E. Beerdsen, B. Smit and D. Dubbeldam, Phys. Rev. Lett. 93 (2004) art. no 248301.

[20] A. G. Bezus, A. V. Kiselev, A. A. Lopatkin and P. Q. Du, J. Chem. Soc., Faraday Trans. II 74 (1978) 367.

[21] S. Calero, D. Dubbeldam, R. Krishna, B. Smit, T. J. H. Vlugt, J. F. Denayer, J. A. Martens and T. L. M. Maesen, J. Am. Chem. Soc. 126 (2004) 11377.

[22] P. Demontis and G. B. Suffritti, Chem. Rev. 97 (1997) 2845.

[23] K. T. Thomson, A. V. McCormick and H. T. Davis, J. Chem. Phys. 112 (2000) 3345.

[24] B. Smit, S. Karaborni and J. I. Siepmann, J. Chem. Phys. 102 (1995) 2126.

[25] J. I. Siepmann, S. Karaborni and B. Smit, Nature 365 (1993) 330.

[26] B. Smit, S. Karaborni and J. I. Siepmann, J. Chem. Phys. 109 (1998) 352.

[27] J. I. Siepmann, M. G. Martin, C. J. Mundy and M. L. Klein, Mol. Phys. 90 (1997) 687.

[28] B. Chen, J. J. Potoff and J. I. Siepmann, J. Phys. Chem. B 105 (2001) 3093.

[29] C. D. Wick, M. G. Martin and J. I. Siepmann, J. Phys. Chem. B 104 (2000) 8008.

[30] B. Chen and J. I. Siepmann, J. Phys. Chem. B 103 (1999) 5370.

[31] M. G. Martin and J. I. Siepmann, J. Phys. Chem. B 103 (1999) 4508.

[32] M. G. Martin and J. I. Siepmann, J. Phys. Chem. B 102 (1998) 2569.

[33] S. K. Nath, F. A. Escobedo and J. J. d. Pablo, J. Chem. Phys. 108 (1998) 9905.

[34] D. Dubbeldam, S. Calero, T. J. H. Vlugt, R. Krishna, T. L. M. Maesen and B. Smit, J. Phys. Chem. B 108 (2004) 12301

[35] P. Pascual, P. Ungerer, B. Tavitian and A. Boutin, J. Phys. Chem. B 108 (2004) 393.

[36] P. Pascual, P. Ungerer, B. Tavitian, P. Pernot and A. Boutin, Phys. Chem. Chem. Phys. 5 (2003) 3684.

[37] Z. Du, T. J. H. Vlugt, B. Smit and G. Manos, AIChE Journal 44 (1998) 1756.

[38] R. Krishna, B. Smit and S. Calero, Chem. Soc. Rev. 31 (2002) 185.

[39] T. J. H. Vlugt, R. Krishna and B. Smit, J. Phys. Chem. B 103 (1999) 1102. 